Running head: SCHOOL BODY IMAGE INTERVENTION

\title{
Happy Being Me in the UK: A controlled evaluation of a school-based body image intervention with pre-adolescent children
}

\begin{abstract}
This study evaluated an adapted version of 'Happy Being Me', a school-based body image intervention, with girls and boys aged 10-11 years. Forty-three children participated in a three-week intervention, and 45 children formed a control group. Both groups completed measures of body satisfaction, risk factors for negative body image, eating behaviors, selfesteem, and intervention topic knowledge, at baseline, post-intervention, and three month follow-up. For girls, participation in the intervention resulted in significant improvements in body satisfaction, appearance-related conversations, appearance comparisons, eating behaviors and intervention topic knowledge at post-intervention, although only the change in body satisfaction was maintained. There was also a significant decrease in internalization of cultural appearance ideals from baseline to follow-up. For boys, participation in the intervention resulted in significant improvements in internalization and appearance comparisons at post-intervention; however, neither of these changes were sustained at followup. There were no improvements in the control group over time.
\end{abstract}

Keywords: Body image; intervention; pre-adolescents; school; body satisfaction 
Running head: SCHOOL BODY IMAGE INTERVENTION

\section{Happy Being Me in the UK: A controlled evaluation of a school-based body image intervention with pre-adolescent children}

Body dissatisfaction develops during childhood and typically $40-50 \%$ of 6-12 year olds report being unhappy with the way they look (Smolak, 2011). Poor body image among children and adolescents can have severe health-related implications, including reduced exercise behavior (Grogan, 2006), unhealthy eating behaviors (Tremblay \& Lariviere, 2009), and mental health problems such as depression (Xie et al., 2010). Therefore there is a need for early intervention to reduce the onset of negative body image among young people (Neumark-Sztainer et al., 2006; Paxton, 2000). In a recent British Government report, early intervention was hailed as an opportunity to "significantly improve mental and physical health, educational attainment and employment" (Allen, 2011, pp. 14).

Due to their ability to reach large groups of young people from differing backgrounds and experiences, many school-based body image interventions have been evaluated, with varying levels of success (Paxton, 2002). These programs have applied a diverse range of methodologies including psycho-education (Baranowski \& Hetherington, 2001), media literacy (Richardson, Paxton, \& Thomson, 2009), and dissonance-based activities (Stice, Shaw, Burton, \& Wade, 2006). Although some of these programs have led to improvements in thin-ideal internalization (e.g., Stice, Chase, Stormer, \& Appel, 2001), disordered eating (e.g., Dalle Grave, De Luca, \& Campello, 2001), and media literacy (e.g., Richardson et al., 2009); many have failed to improve body image (e.g., Dalle Grave et al., 2001; Nicolino, Martz, \& Curtin, 2001; Richardson et al., 2009). Furthermore, despite evidence suggesting that body image concerns often develop in childhood (Cohane \& Pope, 2001; Wood, Becker, \& Thompson, 1996), the majority of school-based interventions have overlooked risk factor reduction in pre-adolescent girls and boys, focusing instead upon adolescents who may have already formed body image concerns. 
Running head: SCHOOL BODY IMAGE INTERVENTION

Stice, Shaw and Marti (2007) conducted a meta-analysis of eating disorder prevention programs, including those that assessed improvements in body image. They found that interventions were more successful with adolescents aged over 15 when compared to those with younger people. It was suggested that younger participants may lack the cognitive ability to participate fully in many of the existing intervention programs. However, in light of the growing evidence for the early onset of negative body image, it seems imperative to develop interventions to address these issues at an earlier age (Holt \& Ricciardelli, 2008).

A comprehensive review of the literature reported that risk factors for negative body image are, in general, either related to individual attributes or external social/environmental factors. It has been proposed that a reduction in such risk factors may 'break' the developmental sequence and thus prevent the onset of negative body image in later life (Jacobi, Hayward, de Zwann, Kraemer, \& Agras, 2004).

\section{'Happy Being Me' and Risk Factors for Negative Body Image}

Body image interventions can be effective in addressing body image concerns among younger adolescents in a school setting. 'Happy Being Me' was designed to target specific risk factors associated with negative body image (internalization of cultural appearance ideals, appearance-related conversations, appearance-related comparisons, and appearancerelated teasing), while also incorporating a self-esteem component (Richardson \& Paxton, 2010). In a previous evaluation with 12-13 year old Australian girls, results revealed a reduction in risk factors for negative body image and disordered eating behavior, an improvement in self-esteem, and an increased awareness of intervention topic; both at postintervention and three month follow-up (Richardson \& Paxton, 2010).

Exposure to media images portraying idealized thin and muscular individuals is commonly associated with the onset of body image concerns (Levine \& Murnen, 2009). In children, research has shown that negative body image can occur when an individual does not 
Running head: SCHOOL BODY IMAGE INTERVENTION

attain their desired, ideal appearance goal (Clark \& Tiggemann, 2008; Jones, Vigfusfottir, \& Lee, 2004). In response, it has been suggested that raising awareness of unrealistic culturalbeauty ideals (Richardson et al., 2009) and reducing internalization of such ideals may help to reduce the onset of negative body image. Evidence suggests that body ideals may differ according to gender. Typically, girls desire a thinner body; whereas boys usually wish to have a muscular physique with minimal body fat (Grogan, 2008; Lawler \& Nixon, 2011). These gender-specific differences require consideration when developing intervention content for girls and boys.

Appearance-related conversations are also a risk factor for body image concerns. Adolescent girls frequently engage in appearance-related conversations with family, peers, and friends (Jones, 2004). Exposure to these conversations has been shown to increase thinideal internalization and body dissatisfaction (Clark \& Tiggeman, 2006). Although fewer studies have investigated the impact of appearance-related conversations on boys' body image concerns, negative appearance-related conversations have been associated with an increased drive for muscularity (Jones \& Crawford, 2006) and higher levels of body image dissatisfaction among adolescent boys (Jones \& Crawford, 2005).

Appearance comparison, defined by Schutz, Paxton and Wertheim (2002) as the tendency to evaluate one's appearance by comparing it with the appearance of another, has been identified as a further risk factor for negative body image. Studies conducted with girls consistently reveal an association between appearance comparison and body dissatisfaction (Keery, van den Berg, \& Thompson, 2004; Mitchell, Petrie, Greenleaf, \& Martin, 2012). The research on social comparisons among boys is less consistent and, in part, highlights the gender specific nature of risk factors for body image disturbance. The majority of evidence suggests that males engage in appearance comparisons and the tendency to engage in such comparisons is positively associated with muscularity dissatisfaction (Smolak \& Stein, 2006). 
Running head: SCHOOL BODY IMAGE INTERVENTION

However, Jones (2004) reported that appearance comparison was not a significant predictor of muscularity-related body dissatisfaction. Research suggests that boys engage in comparisons based specifically on muscularity and these inconsistencies have been attributed in part to the measures used to operationalize constructs such as appearance comparison (McCreary \& Saucier, 2009).

Appearance-related teasing from family, peers, and friends is associated with an increased likelihood of experiencing negative body image among adolescents (Helfert \& Warschburger, 2011; Menzel et al., 2010; Thompson et al., 2007). Parental and sibling teasing is a significant predictor of negative body image among adolescent girls (Keery, Boutelle, van den Berg, \& Thompson, 2005). Notably, a three-year longitudinal study of boys' body dissatisfaction revealed that appearance-related teasing was the only significant risk factor for body dissatisfaction, while other outcome variables of pubertal development, body mass index (BMI), and figure management (i.e., trying to lose weight to look thinner or gain weight to look larger) were not (Barker \& Galambos, 2003).

There is evidence that 'Happy Being Me' is an effective intervention and can address such factors among adolescent girls (Richardson \& Paxton, 2010). However, in light of the evidence demonstrating that boys of all ages are also affected by body image concerns, it would be useful to be able to implement the intervention in mixed-sex schools. Furthermore, although the risk factors associated with the onset of negative body image appear to be broadly similar for girls and boys (Presnell, Bearman, \& Stice, 2004); further exploration of gender-specific risk factors is required. The exclusion of boys from school-based body image interventions is not unique to 'Happy Being Me'. Historically, such interventions have been developed for, and conducted with, girls. In a review of primary school-based interventions by Holt and Ricciardelli (2008), only five of the 13 studies reviewed addressed programs that targeted both girls and boys. 
Running head: SCHOOL BODY IMAGE INTERVENTION

\section{Current Study}

Given the evidence for the early onset of negative body image concerns among girls and boys, and in response to the dearth of literature investigating the effectiveness of intervention programs designed to combat body image concerns in pre-adolescent girls and boys, there is a clear need to develop school-based body image programs that are inclusive and can be implemented successfully in mixed-sex primary (i.e., elementary) schools.

To address this gap, this study aimed to evaluate an adaptation of 'Happy Being Me' among a sample of pre-adolescent girls and boys aged 10-11 years. This is the first known examination of this intervention with children of this age, and the first to include boys. It was hypothesized that girls and boys who completed the intervention would report improved body satisfaction, self-esteem and intervention topic knowledge compared to the control group. It was also hypothesized that children in the intervention group would report reduced internalization of cultural appearance ideals, appearance-related conversations, appearance comparisons, appearance-related teasing, and disordered eating tendencies compared with the control group.

\section{Methods}

\section{Participants}

Following institutional ethical approval and active informed consent from all parents and children, 88 children (46 boys, 42 girls; 100\% Caucasian) aged 10-11 years from two community primary schools located in Southern England volunteered to participate in the study. A quasi-experimental design was utilized; based upon curriculum issues, teachers allocated one class of children from each school to the intervention program and one to the control condition. This design was chosen to avoid classroom disruption and to reduce potential uncontrolled differences between schools that might confound outcomes; a problem noted in the original evaluation of 'Happy Being Me' (Richardson \& Paxton, 2010). 
Running head: SCHOOL BODY IMAGE INTERVENTION

Twenty girls and 23 boys were allocated to the intervention condition $(n=43)$, while 22 girls and 23 boys were allocated to the control group $(n=45)$. All 88 participants completed baseline questionnaires, 85 (96.6\%) completed post-intervention questionnaires and $52(59.1 \%)$ participants completed three month follow-up questionnaires. Four children failed to complete all questionnaire items from the baseline questionnaire, while five children did not complete all items at post-intervention. These data were treated as missing during analyses. The follow-up data collection session fell immediately before the long summer school vacation. Unfortunately, one of the schools was not able to participate in the three month follow-up session due to timetabling constraints at the end of term. Therefore, only data from one school was used to look at longer term intervention effects.

\section{Measures}

All measures were administered immediately before the initial session of the program, immediately post-intervention and at three month follow-up, apart from the demographic questions, which were only completed at baseline. Each measure was assessed, separately for girls and boys, for internal consistency at each time point, as shown in Table 1.

\section{Demographics.}

Participants were asked to report their age and gender in the baseline questionnaire. BMI calculations were computed from self-reported height and weight. Of the 33 children $(38 \%)$ who provided information, the mean BMI was $17.91(S D=3.73)$, ranging from 14.0 to 32.0 .

\section{Body satisfaction.}

An adapted version of the Body Satisfaction Visual Analogue Scale was used to assess body satisfaction (Durkin \& Paxton, 2002). In addition to the one original item "Do you feel satisfied with your body?", an additional four items were constructed for this study (e.g., "Do you feel comfortable with your body shape?", "Do you feel satisfied with your 
Running head: SCHOOL BODY IMAGE INTERVENTION

appearance?", “Do you feel like an attractive person?”, “Do you feel satisfied with your weight?"; Likert-response scale, $1=$ never, $5=$ very often). The additional items were constructed to create a more comprehensive measure of body satisfaction. The items were summed to calculate an overall measure of body satisfaction.

\section{Risk factors for body dissatisfaction and disordered eating.}

Internalization of cultural appearance ideals. The Internalization-General subscale of the Socio-cultural Attitudes Towards Appearance Questionnaire-3 assessed internalization of cultural appearance ideals (Thompson, van den Berg, Roehrig, Guarda, \& Heinberg, 2004). The scale consisted of six items (e.g., "I would like my body to look like people who are on TV"; Likert response scale, 1 = strongly disagree, $5=$ strongly agree). The scale has been shown to produce valid scores in pre-adolescent children (Smolak, Levine, \& Thompson, 2001).

Appearance-related conversations. The Appearance Conversation with Friends subscale of the Factor Loadings for Appearance Culture Among Peers Scale assessed appearance-related conversations (Jones et al., 2004). The scale consisted of five items (e.g., "My friends and I talk about how our bodies look in our clothes"; Likert response scale, 1 = strongly disagree, $5=$ strongly agree). The subscale has been used with girls and boys of a similar age (12-14 years) (Jones et al., 2004).

Appearance comparisons. The Physical Appearance Comparison Scale was included to assess individuals' tendencies to make body comparisons (Thompson, Heinberg, \& Tantleff, 1991). The scale consisted of five items (e.g., "At parties, I compare the way I look to the way other people look"; Likert response scale, 1 = never, 5 = always). This scale has been used with girls and boys of a similar age (11-16 years) (Smolak, Murnen, \& Thompson, 2005; Thompson, Heinberg, Altabe, \& Tantleff-Dunn, 1999).

Appearance-related teasing. The impact of appearance-related teasing was assessed 
Running head: SCHOOL BODY IMAGE INTERVENTION

via the eleven-item Perception of Teasing Scale (Thompson, Cattarin, Fowler, \& Fisher, 1995) (e.g., "If people made fun of you because you were heavy, how upset would you be?"; Likert response scale, $1=$ not upset, $5=$ very upset). This scale has been used with girls and boys of a similar age (6-12 years) (Gardner, Stark, Friedman, \& Jackson, 2000).

\section{Eating behaviors.}

Restrained eating. The Cognitive Restraint subscale of the Three Factor Eating Questionnaire (Stunkard \& Messick, 1985) assessed dietary restraint (e.g., "I do not eat some foods because they make me fat"; Likert response scale, $1=$ never, 5 = very often). Following a pre-intervention focus group session designed to trial program material (see Procedure), two items were removed as they were deemed too complicated for the age group under investigation (e.g. "How frequently do you avoid 'stocking up' on tempting foods?"; “On a scale of 1 to 8 , where 1 means no restraint in eating (eating whatever you want, whenever you want it) and 8 means total restraint (constantly limiting food intake and never 'giving in'), what number would you give yourself?"). This scale has been used across all age groups (de Lauzon et al., 2004).

Emotional eating. The three item Emotional Eating subscale of the Three Factor Eating Questionnaire (Stunkard \& Messick, 1985) assessed emotional eating tendencies (e.g., "When I feel anxious, I find myself eating"; Likert scale response, 1 = never, 5 = very often). This scale has been used across all age groups (de Lauzon et al., 2004).

\section{Self-esteem.}

The Single-Item Self-Esteem Scale (Robins, Hendin, \& Trzesniewski, 2001) was included to assess self-esteem levels (e.g. "I have high self-esteem"; Likert scale response, 1 $=$ never, 5 = very often). Robins et al. (2001) found that the single-item version had convergent validity with children and provided a suitable alternative to the Rosenberg SelfEsteem Scale (Rosenberg, 1965). 
Running head: SCHOOL BODY IMAGE INTERVENTION

\section{Intervention topic knowledge.}

This measure was developed by the intervention authors to specifically assess intervention topic knowledge in relation to 'Happy Being Me' (Richardson \& Paxton, 2010). The original scale consisted of five items (e.g., "The ideal body shape has changed throughout history"; Likert scale response, 1 = strongly disagree, 5 = strongly agree). However, one of the original measure items, "The comparison trap is where people pick out things that they don't like about their appearance and compare those things with other people that they consider better on those things" was removed following the pre-intervention focus group session as it was considered too complicated for the age group under investigation.

\section{Materials}

'Happy Being Me' was originally designed for use with Australian girls aged 12-13 years (For a comprehensive summary of the original program, see Richardson \& Paxton, 2010). Adaptation of the intervention content for pre-adolescent girls and boys in this study therefore required careful thought to address the needs of the differing sample. Table 2 outlines the aims, content and processes used in the adapted program.

Although many of the session aims remained the same and several of the materials used in the original application were retained, some of the content required adaptation. This was to ensure that the program not only addressed body image concerns among girls and boys, engaging both sexes in the material covered, but also that the material was appropriate for the younger target age of 10-11 years. In the first session the intervention group were shown magazine images displaying models prior to, and following, airbrushing manipulation. Where the original program displayed female-only models, the modified program also included images of male models. In addition, where the original program homework exercise presented female-only images throughout history and across cultures, the adapted program also incorporated male images; for example 'Action Man' figurine images were used to 
Running head: SCHOOL BODY IMAGE INTERVENTION

highlight increased muscularity in doll design in recent years.

In the original program, session two involved a female character-only role play based on combating 'fat-talk' and teasing behavior. In the adapted version, a new role-play scenario was developed, including both male and female characters.

In the third session of the original program, girls were shown a film clip, developed as part of the Dove Campaign for Real Beauty (www.dove.co.uk/desp). The clip follows a woman's transformation from an 'ordinary' person to a 'billboard' model, demonstrating image manipulation techniques utilized by the media industry. This clip was moved to the first of the adapted sessions on media literacy. In its place, the third session of the adapted program showed an alternative film clip instead; 'Fabricating Beauty' (Richardson et al., 2009). The clip was chosen as it presented both male and female teenage characters and demonstrated media manipulation techniques, with the aim of ensuring that program material was relevant to both genders.

\section{Procedure}

\section{Focus group testing of intervention material.}

Prior to receiving the intervention, four boys and four girls from the one of the sample schools were invited to participate in two pre-intervention focus groups, separated by gender. The aim of the focus groups was to trial the newly adapted program material and the questionnaire, with the view to identify any problems relating to content suitability for the young, mixed-sex cohort under investigation. Groups were facilitated by the first author and participants were encouraged to talk freely. They were also allowed time to raise objections to the idea of mixed-sex classes, however, no objections were raised.

Each group were provided with sample handout materials to be used within the main intervention and were asked to discuss their thoughts and feelings relating to the images displayed. These tasks were used to gauge the children's reactions and responses to the 
Running head: SCHOOL BODY IMAGE INTERVENTION

material designed for use in the main intervention, in the hope of identifying any iatrogenic effects prior to facilitation of the main program. Children were also asked to complete sample items taken from each of the measures utilized in the main questionnaire, and to discuss their thoughts and feelings relating to those items. Only items that were easy to interpret and age appropriate were included in the final questionnaire.

\section{'Happy Being Me' intervention.}

Immediately prior to the first intervention session, both the intervention and control groups were asked to complete the baseline questionnaire.

Children in the intervention condition participated in three, one hour sessions facilitated by the first author, with one week between each session. The control group received their standard Personal, Social, Health and Economic (PSHE) lessons, facilitated by their usual class teacher. These lessons are a part of the national curriculum and are designed to help children develop their knowledge, understanding and skills of real life issues and experiences, with the aim of promoting general wellbeing and community cohesion.

Immediately following the final intervention session, both groups were asked to complete the post-intervention questionnaire. At three months post-intervention both groups completed a follow-up questionnaire.

\section{Results}

\section{Demographic Equivalence of the Groups}

For girls, at baseline there was no difference in BMI $\left(t=0.41, d f=13, p=.69, \eta^{2}=\right.$ 0.01 ; Intervention $M=20.17, S D=6.31$; Control $M=19.11, S D=3.62)$, or age $(t=1.24, d f=$ $40, p=.90, \eta^{2}=0.02 ;$ Intervention $M=10.70, S D=0.47 ;$ Control $\left.M=10.68, S D=0.48\right)$ between the intervention and control conditions. For boys, at baseline there was no difference in BMI $\left(t=0.61, d f=16, p=.55, \eta^{2}=0.02\right.$; Intervention $M=16.30, S D=1.95$; Control $M=$ $16.88, S D=2.03)$, or age $\left(t=1.48, d f=44, p=.15, \eta^{2}=0.02\right.$; Intervention: $M=10.39, S D=$ 
Running head: SCHOOL BODY IMAGE INTERVENTION

0.50; Control $M=10.61, S D=0.50)$ between the intervention and control conditions.

\section{Pre-intervention Outcome Equivalence of the Groups}

Multivariate analysis of variance (MANOVA) to identify baseline differences between intervention and control conditions on the outcome variables demonstrated no significant multivariate effect for the relationship for girls, Hotelling's $T(9,27)=0.31, p=$ .53 , partial $\eta^{2}=.23$; or boys, Hotelling's $T(9,34)=0.27, p=.46$, partial $\eta^{2}=.21$.

\section{Gender Differences in Study Variables}

Analyses to identify baseline differences between girls and boys on the outcome variables revealed a significant multivariate effect for the relationship, Hotelling's $T(9,71)=$ $0.37, p=.01$, partial $\eta^{2}=.27$. As shown in Table 3 , univariate results for this relationship demonstrated a significant effect for internalization, $F(1,79)=3.61, p=.04$, partial $\eta^{2}=.04$; appearance-related comparison, $F(1,79)=5.51, p=.02$, partial $\eta^{2}=.07$; appearance-related teasing, $F(1,79)=14.94, p=.01$, partial $\eta^{2}=.01$; and self-esteem, $F(1,79)=6.94, p=.01$, partial $\eta^{2}=.08$

\section{Examination of the Effects of the Intervention Program}

To examine the effects of the intervention, analyses were conducted separately for girls and boys. Mixed between-within MANOVA, with intervention condition and time as the independent variables, were conducted to identify any changes in the outcome variables in the intervention and control conditions from baseline to post-intervention. In response to one school not being available for follow-up data collection and therefore a smaller sample at three month follow-up, $3 \times 2$ analyses of variance (ANOVA) were conducted separately to examine the longer term impact of the intervention. Table 1 provides means and standard deviations on the outcome variables for the intervention and control conditions.

\section{Girls' baseline to post-intervention analyses.}

At the multivariate level, there was a significant time $\mathrm{x}$ condition interaction, $F(9,22)$ 
Running head: SCHOOL BODY IMAGE INTERVENTION

$=4.08, p=.01 ;$ Wilks $^{\prime} \lambda=0.38$. As shown in Table 4 , at the univariate level the time $\mathrm{x}$ condition interactions were significant for girls' body satisfaction, appearance-related conversations, appearance-related comparisons, restrained eating, emotional eating, and intervention topic knowledge.

Follow-up paired comparisons revealed that girls in the intervention condition reported significant improvements from baseline to post-intervention in body satisfaction $\left(M_{\text {diff }}=-2.00, p=.03, d=0.62^{1}\right)$. They also reported a significant reduction in appearancerelated conversations $\left(M_{\text {diff }}=3.12, p=.009, d=0.67\right)$, appearance-related comparisons $\left(M_{\text {diff }}\right.$ $=2.53, p=.001, d=0.92)$, restrained eating $\left(M_{\text {diff }}=1.59, p=.02, d=0.48\right)$, and emotional eating scores $\left(M_{\text {diff }}=2.24, p=.001, d=0.92\right)$ at post-intervention. There were also significant improvements in intervention topic knowledge scores $\left(M_{\text {diff }}=-3.29, p=.01, d=\right.$ 0.30). There were no significant changes in internalization, self-esteem and appearancerelated teasing from baseline to post-intervention among the intervention group girls.

In the control condition, univariate analysis revealed that girls' body satisfaction scores significantly decreased $\left(M_{\text {diff }}=1.72, p=.012, d=0.46\right)$ and emotional eating significantly increased $\left(M_{\text {diff }}=-1.56, p=.048, d=-0.40\right)$ at post-intervention. There were no significant changes on the other outcome variables for the control group from baseline to post-intervention.

\section{Girls' baseline to three month follow-up analyses.}

To examine the longer term impact of the intervention 3 (baseline, immediate postintervention, follow-up) x 2 (intervention, control) ANOVAs were conducted on the outcome variables that changed immediately post-intervention. Although, there were a smaller group of girls who completed the three month follow-up questionnaire, minimum criteria for cell size within the ANOVAs were met (Wilson Van Voorhis \& Morgan, 2007). There was a

\footnotetext{
${ }^{1}$ Effect sizes were calculated for within subjects design correcting for dependence among means.
} 
Running head: SCHOOL BODY IMAGE INTERVENTION

significant interaction effect between time and condition for body satisfaction, $F(2,36)=$ $3.39, p=.045$, partial $\eta^{2}=.16$. Follow-up analysis revealed a significant change in body satisfaction for girls in the intervention group, $F(2,18)=8.33, p=.003$, partial $\eta^{2}=.48$. Paired comparisons revealed a significant increase in body satisfaction from baseline to postintervention $\left(M_{\text {diff }}=-3.10, p=.04, d=-0.83\right)$, that was maintained at three month follow up $\left(M_{\text {diff }}=-5.20 p=.005, d=-1.23\right)$. There was no significant change in body satisfaction for the control group, $F(2,18)=3.13, p=.07$, partial $\eta^{2}=.26$.

There was a significant time $\mathrm{x}$ condition interaction for emotional eating, $F(2,34)=$ $3.25, p=.05$, partial $\eta^{2}=.16$. However, there was no significant effect of time in the intervention group, $F(2,16)=2.45, p=.12$, partial $\eta^{2}=.24$, and the interaction effect was driven by changes in the control group, $F(2,16)=4.01, p=.04$, partial $\eta^{2}=.31$. Paired comparisons revealed, mirroring the previous findings, emotional eating increased from baseline to post-intervention in the control group $\left(M_{\text {diff }}=-2.60 p=.05, d=-0.67\right)$. However, this change was no longer evident in analysis of baseline to three month follow up data $\left(M_{\text {diff }}\right.$ $=0.60 p=.60, d=0.09)$.

The time $\mathrm{x}$ condition interaction for intervention topic knowledge was significant, $F(2,36)=3.43, p=.04$, partial $\eta^{2}=.16$. In the intervention condition, the main effect for time approached significance, $F(2,18)=3.07, p=.07$, partial $\eta^{2}=.25$, with a significant increase in topic knowledge from baseline to post-intervention $\left(M_{\text {diff }}=-4.10, p=.04, d=-\right.$ 0.93). However, this change was no longer evident at follow up $\left(M_{\text {diff }}=-1.60, p=.47, d=-\right.$ 0.24). The effect of time was not significant in the control group, $F(2,18)=2.81, p=.09$, partial $\eta^{2}=.24$, and there were no significant differences on the paired comparisons $(p$ 's $>.05)$.

There were no significant time $\mathrm{x}$ condition interactions for appearance conversations, $F(2,34)=0.59, p=.56$, partial $\eta^{2}=.03$, appearance comparisons, $F(2,28)=0.56, p=.58$, 
partial $\eta^{2}=.04$, or restrained eating, $F(2,36)=1.40, p=.26$, partial $\eta^{2}=.08$, indicating that there was no change across time on these variables for either condition.

\section{Boys' baseline to post-intervention.}

There was a significant time $\mathrm{x}$ condition interaction at the multivariate level for boys, $F(9,32)=2.32, p=.04 ;$ Wilks $^{\prime} \lambda=0.61$. The univariate time $\mathrm{x}$ condition interactions reported in Table 4 show that there was a significant change over time across conditions for boys' internalization and appearance-related conversations.

Follow-up paired comparisons revealed that boys in the intervention condition reported significantly lower levels of internalization of cultural appearance ideals $\left(M_{\text {diff }}=\right.$ $3.36, p=.004, d=0.62)$ and appearance-related conversations $\left(M_{\text {diff }}=3.36, p=.004, d=\right.$ $0.63)$ immediately post-intervention. There were no significant changes on these variables in the control condition (cultural appearance internalization $M_{\text {diff }}=-0.61, p=.58$; appearancerelated conversations $\left.M_{\text {diff }}=1.30, p=.09\right)$. Neither the intervention or control group showed any change from baseline to post-intervention on the remaining outcome variables.

\section{Boys' baseline to three month follow-up.}

In the smaller sample of boys who completed the follow up questionnaire, there was no significant time $\mathrm{x}$ condition interaction in 3 (baseline, immediate post-intervention, follow up) $\mathrm{x} 2$ (intervention, control) ANOVAs for cultural appearance internalization, $F(2,44)=$ $1.12, p=.34$, partial $\eta^{2}=.05$, or appearance-related conversations, $F(2,42)=0.84, p=.44$, partial $\eta^{2}=.04$

\section{Discussion}

This study evaluated the impact of an adapted version of 'Happy Being Me', a schoolbased body image intervention. The intervention used an etiologic approach with a mixed-sex cohort of 10-11 year old children. This controlled study was the first known application of 'Happy Being Me' (Richardson \& Paxton, 2010) with a pre-adolescent cohort, and was novel 
Running head: SCHOOL BODY IMAGE INTERVENTION

in its inclusion of girls and boys.

Analyses of the girls' intervention group scores revealed an immediate postintervention improvement on body satisfaction, appearance-related comparisons, appearancerelated conversations, restrained eating, emotional eating, and intervention topic knowledge. However, the study did not identify any post-intervention effects on appearance-related teasing, internalization of cultural appearance ideals, or self-esteem. Further, only improvements in body satisfaction were maintained at three month follow-up for the girls.

Boys participating in the intervention reported immediate improvements in internalization of cultural appearance ideals and appearance-related conversations but were not maintained at three month follow-up. None of the other outcome variables were impacted by the intervention. These findings suggest, perhaps not surprisingly, that the intervention was not as effective for boys as it was for girls. Studies of body image interventions among adolescent boys frequently fail to find intervention effects (e.g., McCabe, Ricciardelli, \& Karantzas, 2010). However, it should be noted that female body image interventions have received far more attention than male body image interventions, which are still in their relative infancy. Therefore, even short-term changes in risk factors for negative body image identified by this study are encouraging. Furthermore, these results suggest that 'Happy Being Me' could be implemented in mixed-sex settings without doing harm to boys. Nevertheless, this program needs refinement to improve its effectiveness among preadolescent boys.

The immediate positive changes reported for appearance-related conversations in girls and boys are consistent with the findings of Richardson and Paxton (2010) for adolescent girls, and are promising considering the reported high prevalence of appearance conversations among children (Jones, 2004). Interestingly, the measure used to assess appearance-related conversations was focused on weight-related appearance (Jones, 2004), a 
Running head: SCHOOL BODY IMAGE INTERVENTION

topic that may be less relevant to boys than girls (Grogan, 2008; Lawler \& Nixon, 2011). The current findings nevertheless suggest that pre-adolescent boys also engage in weight-related appearance-related conversations, and perhaps more importantly, demonstrate that the adaptation of 'Happy Being Me' may help to combat these issues in the short-term. However, future development of 'Happy Being Me' may benefit from a specific focus on boys' body image concerns, including additional measures that specifically assess muscularity concerns.

In line with the original findings (Richardson \& Paxton, 2010), girls' appearancerelated comparison scores and unhealthy eating behaviors were significantly reduced at postintervention. Vohs, Heatherton and Herrin (2001) argued that eating pathology does not generally emerge, or increase notably, during pre-adolescence; rather, it tends to present during adolescence (Lewinsohn, Striegel-Moore, \& Seeley, 2000). However, our findings provide evidence for the presence of restrained eating behavior in pre-adolescents girls and a significant improvement in eating scores from pre- to post-intervention. Considering that the original program did not set out to address eating behaviors, these findings are particularly promising, especially when the majority of school-based interventions have failed to reduce disordered-eating behaviors (Dalle Grave et al., 2001; Richardson et al., 2009). Further adaptation of the program to include follow-up exercises may help to sustain these effects over time.

In contrast, boys' appearance-related comparison scores and eating behaviors were not affected by the intervention. Furthermore, the intervention did not have the desired impact upon self-esteem for girls or boys. Although the finding for self-esteem is at odds with the original application, it is not unusual in the wider context of the previous school-based body image intervention literature (McVey et al., 2003; Steiner-Adair et al., 2002). In addition, self-esteem tends to be classified as a non-specific risk factor for body dissatisfaction and therefore may be more difficult to change in a brief intervention (Mann, 
Running head: SCHOOL BODY IMAGE INTERVENTION

Hosman, Schaalma, \& de Vries, 2004).

It is important to note that the positive intervention effects found here contradict concerns that exposure to media images during intervention programs may actually contribute to the development of negative body image (Durkin, Paxton, \& Sorbello, 2007). The results also indicate that this age group of girls and boys can benefit from body image interventions.

Generally, the lack of maintenance of the intervention effects contrasts the original findings (Richardson \& Paxton, 2010), and is concerning. There was a relatively small sample size at follow-up and it may be that there was insufficient power to detect effects that were maintained. However, the results may also suggest that this age group require additional sessions to achieve more stable change on risk factors for negative body image and disordered eating behavior. Children are regularly exposed to appearance-related pressures (Tremblay \& Lariviere, 2009) and therefore, it may be that providing three 50 minute sessions is insufficient to counter the socially normative behavior described by Jones and colleagues as an "appearance culture" (2004, pp. 323).

There are a number of limitations to the current study. Clearly, the small sample at follow-up is problematic and the research needs to be replicated with a larger sample of preadolescents. For instance, although not statistically significant, the moderate effect sizes for changes in girls' body satisfaction, restrained eating, emotional eating, and intervention topic knowledge scores over time indicate limited statistical power in the follow-up analyses. Power analysis revealed that 128 participants would be required to detect an effect size of 0.80. Another limitation of the study is the non-random allocation of participants to condition due to the teachers' timetabling concerns. In addition, as children were allocated to each condition by class, rather than by school, there may have been spill-over effects. Although not ideal, constraints on condition allocation are typical of applied research. In this case, 
Running head: SCHOOL BODY IMAGE INTERVENTION

concerns about lack of randomization may be reduced by the lack of baseline differences between conditions on the study outcome variables and participant demographics. Finally, it is important to identify that the measures used to assess body image concerns may not have been appropriate for use with a pre-adolescent mixed-sex cohort. For example, although some of the questionnaire items were adapted for use with a pre-adolescent sample, the small alpha values identified for some constructs suggest that further exploration of the measures is required. Furthermore, while the risk-factors for negative body image are well-documented for girls and boys, it is possible that the gender-specific nuances of such risk factors were not adequately captured, as many of the measures utilized in this study were originally designed for use with girls. This may have resulted in a failure to identify male-specific tendencies, such as drive for muscularity (McCreary \& Saucier, 2009), and as a result the differential effects of the intervention for girls and boys should be interpreted in light of these measurement issues. Future application of this intervention may therefore benefit from the measurement of gender-specific risk factors.

Despite its acknowledged shortcomings, the current study adds to the limited evidence-base for pre-adolescent, mixed-sex, school-based body image interventions. This study revealed some positive intervention effects in a mixed-sex school environment and it highlights the potential for future development and application of 'Happy Being Me' (Richardson \& Paxton, 2010). This study also suggests that school-based interventions with a pre-adolescent cohort can be beneficial, and it adds to the growing support for the development of intervention programs designed specifically for pre-adolescent children.

\section{Acknowledgements}


Running head: SCHOOL BODY IMAGE INTERVENTION

The authors would like to thank Susan Paxton for sharing the original 'Happy Being Me' program materials. Thank you also to the school staff and students that were involved in this research.

\section{References}

Allen, G. (2011). Early intervention: the next steps. London: Cabinet Office.

Baranowski, M. J., \& Hetherington, M. M. (2001). Testing the efficacy of an eating disorder prevention program. International Journal of Eating Disorders, 29, 119-124.

Barker, E. T., \& Galambos, N. L. (2003). Body dissatisfaction of adolescent girls and boys: Risk and resource factors. Journal of Early Adolescence, 23, 141-165.

Clark, L. S., \& Tiggemann, M. (2006). Appearance culture in 9- to 12-year-old girls: Media and peer influences on body dissatisfaction. Journal of Social Development, 15, 628643.

Clark, L. S., \& Tiggemann, M. (2008). Sociocultural and individual psychological predictors of body image in young girls: A prospective study. Developmental Psychology, 44, $1124-1134$.

Cohane, G. H., \& Pope, H. G. (2001). Body image in boys: A review of the literature. International Journal of Eating Disorders, 29, 373-379.

Dalle Grave, R., De Luca, L., \& Campello, G. (2001). Middle school primary prevention program for eating disorders: A controlled study with a twelve-month follow-up. Eating Disorder Review, 9, 327-337.

de Lauzon, B., Romon, M., Deschamps, V., Lafay, L., Borys, J-M., Karlsson, J., Ducimetière, P., Aline Charles, M., \& Fleurbaix Laventie Ville Sante (FLVS) Study Group. (2004). The three-factor eating questionnaire-R18 is able to distinguish among different eating patterns in a general population. Journal of Nutrition, 134, 2372-2380.

Durkin, S. J., \& Paxton, S. J. (2002). Predictors of vulnerability to reduced body image 
Running head: SCHOOL BODY IMAGE INTERVENTION

satisfaction and psychological wellbeing in response to exposure to idealized female media images in adolescent girls. Journal of Psychosomatic Research, 53, 995-1005.

Durkin, S. J., Paxton, S. J., \& Sorbello, M. (2007). An integrative model of the impact of exposure to idealized female images on adolescent girls' body satisfaction. Journal of Applied Social Psychology, 37, 1092-1117.

Gardner, R. M., Stark, K., Friedman, B. N., \& Jackson, N. A. (2000). Predictors of eating disorder scores in children ages 6 through 14: A longitudinal study. Journal of Psychosomatic Research, 49, 199-205.

Grogan, S. (2006). Body image and health: Contemporary perspectives. Journal of Health Psychology, 11, 523-530.

Grogan, S. (2008). Body Image. Understanding body dissatisfaction in men, women, and children (2nd ed.). London: Routledge.

Helfert, S., \& Warschburger, P. (2011). A prospective study on the impact of peer and parental pressure on body dissatisfaction in adolescent girls and boys. Body Image, 8 , 101-109.

Holt, K. E., \& Ricciardelli, L. A. (2008). Weight concerns among elementary school children: A review of prevention programs. Body Image, 5, 233-243.

Jacobi, C., Hayward, C., de Zwann, M., Kraemer, H. C., \& Agras, W. S. (2004). Coming to terms with risk factors for eating disorders: Application of risk terminology and suggestions for a general taxonomy. Psychology Bulletin, 130, 19-65.

Jones, D. C. (2004). Body image among adolescent girls and boys: A longitudinal study. Journal of Developmental Psychology, 40, 823-835.

Jones, D. C., \& Crawford, J. K. (2005). Adolescent boys and body image: Weight and muscularity concerns as dual pathways to body dissatisfaction. Journal of Youth and Adolescence, 34, 629-636. 
Running head: SCHOOL BODY IMAGE INTERVENTION

Jones, D. C., \& Crawford, J. K. (2006). The peer appearance culture during adolescence: Gender and body mass variations. Journal of Youth and Adolescence, 35, 257-269.

Jones, D. C., Vigfusfottir, T. H., \& Lee, Y. (2004). Body image and the appearance culture among adolescent girls and boys: An examination of friend conversations, peer criticism, appearance magazines, and the internalization of appearance ideals. Journal of Adolescent Research, 19, 323-339.

Keery, H., Boutelle, K., van den Berg, P., \& Thompson, J. K. (2005). The impact of appearance related teasing by family members. Journal of Adolescent Health, 37, $120-127$.

Keery, H., van den Berg, P., \& Thompson, J. K. (2004). An evaluation of the tripartite influence model on body dissatisfaction and eating disturbance with adolescent girls. Body Image, 1, 237-251.

Krayer, A., Ingledew, D. K., \& Iphofen, R. (2008). Social comparison and body image in adolescence: A grounded theory approach. Health Education Research, 23, 892-903.

Lawler, M., \& Nixon, E. (2011). Body dissatisfaction among adolescent boys and girls: The effects of body mass, peer appearance culture and internalization of appearance ideals. Journal of Youth and Adolescence, 40, 59-71.

Levine, M. P., \& Murnen, S. K. (2009). "Everybody knows that mass media are/are not [pick one] a cause of eating disorders". A critical review of evidence for a causal link between media, negative body image and disordered eating in females. Journal of Social and Clinical Psychology, 28, 9-42.

Lewinsohn, P. M., Striegel-Moore, R. H., \& Seeley, J. R. (2000). Epidemiology and natural course of eating disorders in young women from adolescence to young adulthood. Journal of the American Academy of Child and Adolescent Psychiatry, 39, 12841292. 
Running head: SCHOOL BODY IMAGE INTERVENTION

Mann, M., Hosman, C. M. H., Schaalma, H. P., \& de Vries, N. K. (2004). Self-esteem in a broad-spectrum approach for mental health promotion. Health Education Research, $19,357-372$.

McCabe, M. P., Ricciardelli, L. A., \& Karantzas, G. (2010). Impact of a healthy body image program among adolescent boys on body image, negative affect, and body change strategies. Body Image, 7, 117-123.

McCreary, D. R., \& Saucier, D. M. (2009). Drive for muscularity, body comparison, and social physique anxiety in men and women. Body Image, 6, 24-30.

McVey, G. L., Lieberman, M., Voorberg, N., Wardrope, D., Blackmore, E., \& Tweed, S. (2003). Replication of a peer support program designed to prevent disordered eating: Is a life skills approach sufficient for all middle school students? Journal of Eating Disorders, 11, 187-195.

Menzel, J. E., Schaefer, L. M., Burke, N. L., Mayhew, L. L., Brannick, M. T., \& Thompson, J. K. (2010). Appearance-related teasing, body dissatisfaction, and disordered eating: A meta-analysis. Body Image, 7, 261-270.

Mitchell, S. H., Petrie, T. A., Greenleaf, C. A., \& Martin, S. B. (2012). Moderators of the internalization-body dissatisfaction relationship in middle school girls. Body Image, 9, 431-440.

Neumark-Sztainer, D., Levine, M. P., Paxton, S., Smolak, L., Piran, N., \& Wertheim, E. H. (2006). Prevention of body dissatisfaction and disordered eating: What next? Eating Disorders, 14, 265-285.

Nicolino, J. C., Martz, D. M., \& Curtin, L. (2001). Evaluation of a cognitive-behavioral therapy intervention to improve body image and decrease dieting in college women. Eating Behaviors, 2, 353-362.

Paxton, S. J. (2000). Body image dissatisfaction, extreme weight loss behaviors: Suitable 
Running head: SCHOOL BODY IMAGE INTERVENTION

targets for public health concern? Health Promotion Journal of Australia, 10, 15-19.

Paxton, S. J. (2002). Research review of body image programs: An overview of body image dissatisfaction prevention interventions. Melbourne, Victoria: Rural and Regional Health and Aged Care Services Division.

Presnell, K., Bearman, S. K., \& Stice, E. (2004). Risk factors for body dissatisfaction in adolescent boys and girls: A prospective study. International Journal of Eating Disorders, 36, 389-401.

Richardson, S. M., \& Paxton, S. J. (2010). An evaluation of a body image intervention based on risk factors for body dissatisfaction: A controlled study with adolescent girls. International Journal of Eating Disorders, 43, 112-122.

Richardson, S. M., Paxton, S. J., \& Thomson, J. S. (2009). Is BodyThink an efficacious body image and self-esteem program? A controlled evaluation with adolescents. Body Image, 6, 75-82.

Robins, R. W., Hendin, H. M., \& Trzesniewski, K. H. (2001). Measuring global self-esteem: Construct validation of a single-item measure of the Rosenberg self-esteem scale. Personality and Social Psychology Bulletin, 27, 151-161.

Rosenberg, M. (1965). Society and the adolescent self-image. Princeton, NJ: Princeton University Press.

Schutz, H. K., Paxton, S. J., \& Wertheim, E. H. (2002). Investigation of body comparison among adolescent girls. Journal of Applied Social Psychology, 32, 1906-1937.

Smolak, L. (2011). Body image development in childhood. In Cash, T., \& Smolak, L. (Eds). Body image: A handbook of science, practice and prevention (pp. 67-75). New York: Guildford Press.

Smolak, L., Levine, M. P., \& Thompson, J. K. (2001). The use of the Sociocultural Attitudes towards Appearance Questionnaire with middle school boys and girls. International 
Running head: SCHOOL BODY IMAGE INTERVENTION

Journal of Eating Disorders, 29, 216-223.

Smolak, L., Murnen, S. K., \& Thompson, J. K. (2005). Sociocultural influences and muscle building in adolescent boys. Psychology of Men and Masculinity, 6, 227-239.

Smolak, L., \& Stein, J. A. (2006). The relationship of drive for muscularity to sociocultural factors, self-esteem, physical attributes gender role, and social comparison in middle school boys. Body Image, 3, 121-129.

Steiner-Adair, C., Sjostrom, L., Franko, D. L., Pai, S., Tucker, R., Becker, A. E., \& Herzog, D. B. (2002). Primary prevention of risk factors for eating disorders in adolescent girls: Learning from practice. International Journal of Eating Disorders, 32, 401-411.

Stice, E., Chase, A., Stormer, S., \& Appel, A. (2001). A randomized trial of a dissonance based eating disorder prevention program. International Journal of Eating Disorders, $29,247-262$.

Stice, E., Shaw, H., Burton, E., \& Wade, E. (2006). Dissonance and healthy weight eating disorder prevention programs: A randomized efficacy trial. Journal of Consulting and Clinical Psychology, 74, 263-275.

Stice, E., Shaw, H., \& Marti, N. (2007). A meta-analytic review of eating disorder prevention programs: Encouraging findings. Annual Review of Clinical Psychology, 3, 207-231.

Stunkard, A. J., \& Messick, S. (1985). The three-factor eating questionnaire to measure dietary restraint, disinhibition and hunger. Journal of Psychosomatic Research, 29, 79-83.

Thompson, J. K., Cattarin, J., Fowler, B., \& Fisher, E. (1994). The perception of teasing scale (POTS): A revision and extension of the physical appearance related teasing scale (PARTS). Journal of Personality and Assessment, 65, 157. 
Running head: SCHOOL BODY IMAGE INTERVENTION

Thompson, J. K., Heinberg, L., Altabe, M., \& Tantleff-Dunn, S. (1999). Exacting beauty: Theory, assessment, and treatment of body image disturbance. Washington, DC: American Psychological Association.

Thompson, J. K., Heinberg, L. J., \& Tantleff, S. (1991). The physical appearance comparison scale (PACS). The Behavior Therapist, 14, 137.

Thompson, J. K., Shroff, H., Herbozo, S., Cafri, C., Rodriguez, J., \& Rodriguez, M. (2007). Relations among multiple peer influences, body dissatisfaction, eating disturbance, and self-esteem: A comparison of average weight, at risk of overweight, and overweight adolescent girls. Journal of Pediatric Psychology, 32, 24-29.

Thompson, J. K., van den Berg, P., Roehrig, M., Guarda, A. S., \& Heinberg, L. J. (2004). The sociocultural attitudes toward appearance questionnaire (SATAQ-3): Development and validation. International Journal of Eating Disorders, 35, 293-304.

Tremblay, L., \& Lariviere, M. (2009). The influences of puberty onset, body mass index, and pressure to be thin on disordered eating behaviors in children and adolescents. Journal of Eating Behaviors, 10, 75-83.

Vohs, K. D., Heatherton, T. F., \& Herrin, M. (2001). Disordered eating and the transition to college: A prospective study. International Journal of Eating Disorders, 29, 280-288.

Wilson Van Voorhis, C. R., \& Morgan, B. L. (2007). Understanding power and rules of thumb for determining sample sizes. Tutorials in Quantitative Methods for Psychology, 3, 43-50.

Wood, K. C., Becker, J. A., \& Thompson, J. K. (1996). Body image dissatisfaction in preadolescent children. Journal of Applied Developmental Psychology, 17, 85-100.

Xie, B., Unger, J. B., Gallaher, B., Anderson Johnson, C., Wu, Q., \& Chu, C-P. (2010). Overweight, body image, and depression in Asian and Hispanic adolescents. American Journal of Health Behavior, 34, 476-488. 


\section{Running head: SCHOOL BODY IMAGE INTERVENTION}

Table 1

Descriptive Statistics

\begin{tabular}{|c|c|c|c|c|c|c|c|c|c|c|}
\hline \multirow[b]{2}{*}{ Girls } & \multirow[b]{2}{*}{ Scale Range } & \multicolumn{3}{|c|}{ Intervention } & \multicolumn{3}{|c|}{ Control } & \multicolumn{3}{|c|}{ Internal Consistency } \\
\hline & & T1 Mean $(S D)$ & T2 Mean $(S D)$ & T3 Mean $(S D)$ & T1 Mean $(S D)$ & T2 Mean $(S D)$ & T3 Mean $(S D)$ & $\alpha$ & $\alpha$ & $\alpha$ \\
\hline Body satisfaction (a) & $4-20$ & $11.60(3.03)$ & $13.63(2.93)$ & $15.09(3.21)$ & $12.77(3.85)$ & $11.1(3.87)$ & $13.64(1.91)$ & .79 & .89 & .81 \\
\hline Cultural appearance internalization (b) & $6-30$ & $17.35(5.55)$ & $15.94(6.02)$ & $10.91(5.01)$ & $17.64(5.36)$ & $17.30(7.1)$ & $11.73(3.17)$ & .80 & .92 & .91 \\
\hline Appearance-related conversation (b) & $5-25$ & $12.89(5.90)$ & $10.22(4.40)$ & $8.18(3.09)$ & $12.95(5.51)$ & $12.52(5.82)$ & $10.18(4.90)$ & .89 & .89 & .85 \\
\hline Appearance-related comparison (b) & $5-25$ & $14.33(3.91)$ & $11.89(4.08)$ & $9.30(2.83)$ & $13.48(3.91)$ & $12.71(4.14)$ & $10.73(3.47)$ & .60 & .84 & .85 \\
\hline Appearance-related teasing $(\mathrm{b})$ & $11-55$ & $45.60(11.96)$ & $39.11(10.48)$ & $36.18(10.16)$ & $42.27(7.65)$ & $41.43(7.30)$ & $44.82(6.52)$ & .52 & .87 & .89 \\
\hline Restrained eating (b) & $4-20$ & $9.25(4.84)$ & $8.11(4.48)$ & $4.50(.71)$ & $9.00(3.73)$ & $9.76(5.22)$ & $8.09(4.09)$ & .91 & .92 & .86 \\
\hline Emotional eating (b) & $3-15$ & $6.65(3.25)$ & $4.84(2.19)$ & $3.70(1.34)$ & $5.00(2.31)$ & $6.24(3.46)$ & $5.36(2.54)$ & .82 & .81 & .77 \\
\hline Self-esteem (a) & $1-5$ & $2.90(0.91)$ & $3.33(1.08)$ & $3.80(.79)$ & $2.68(1.25)$ & $2.86(1.35)$ & $3.36(1.50)$ & - & - & - \\
\hline \multirow[t]{2}{*}{ Intervention topic knowledge (a) } & $4-20$ & $13.75(4.30)$ & $16.58(2.85)$ & $15.55(2.73)$ & $14.32(2.72)$ & $14.24(2.51)$ & $16.00(1.61)$ & .70 & .55 & .33 \\
\hline & & \multicolumn{3}{|c|}{ Intervention } & \multicolumn{3}{|c|}{ Control } & \multicolumn{3}{|c|}{ Internal Consistency } \\
\hline Boys & Scale Range & T1 Mean $(S D)$ & T2 Mean $(S D)$ & T3 Mean $(S D)$ & T1 Mean $(S D)$ & T2 Mean $(S D)$ & T3 Mean $(S D)$ & $\alpha$ & $\alpha$ & $\alpha$ \\
\hline Body satisfaction (a) & $4-20$ & $13.52(3.26)$ & $14.00(3.28)$ & $13.93(3.49)$ & $13.48(4.72)$ & $13.48(3.52)$ & $13.00(4.03)$ & .78 & .63 & .64 \\
\hline Cultural appearance internalization (b) & $6-30$ & $17.04(5.86)$ & $14.00(5.84)$ & $13.14(4.65)$ & $12.78(5.93)$ & $13.39(6.40)$ & $10.73(5.44)$ & .82 & .97 & .84 \\
\hline Appearance-related conversation (b) & $5-25$ & $12.35(6.09)$ & $9.27(5.57)$ & $10.85(5.23)$ & $10.35(6.61)$ & $9.09(5.40)$ & $6.45(3.01)$ & .91 & .82 & .86 \\
\hline Appearance-related comparison (b) & $5-25$ & $12.30(3.84)$ & $10.86(3.85)$ & $11.23(2.92)$ & $11.36(4.59)$ & $10.87(4.03)$ & $8.91(2.12)$ & .78 & .75 & .73 \\
\hline Appearance-related teasing $(\mathrm{b})$ & $11-55$ & $35.78(10.84)$ & $32.09(12.17)$ & $41.14(9.99)$ & $34.57(11.92)$ & $29.91(13.39)$ & $25.20(11.26)$ & .90 & .94 & .95 \\
\hline Restrained eating (b) & $4-20$ & $7.77(3.46)$ & $6.52(3.04)$ & $8.08(3.99)$ & $8.00(4.49)$ & $7.57(3.80)$ & $5.82(2.99)$ & .87 & .84 & .89 \\
\hline Emotional eating (b) & $3-15$ & $5.70(2.27)$ & $5.09(2.72)$ & $6.46(3.62)$ & $5.96(4.05)$ & $5.30(2.69)$ & $3.73(1.68)$ & .79 & .83 & .95 \\
\hline Self-esteem (a) & $1-5$ & $3.57(1.20)$ & $3.55(1.34)$ & $3.36(1.34)$ & $3.43(1.34)$ & $3.09(1.35)$ & $2.64(1.12)$ & - & - & - \\
\hline Intervention topic knowledge (a) & $4-20$ & $14.39(3.33)$ & $16.00(2.51)$ & $16.36(1.39)$ & $12.61(4.47)$ & $13.22(4.17)$ & $14.73(2.20)$ & .78 & .62 & .61 \\
\hline
\end{tabular}




\section{Running head: SCHOOL BODY IMAGE INTERVENTION}

Table 2

An outline of the aims and activities included in the adapted 'Happy Being Me' program

\begin{tabular}{ccc}
\hline Aims & Content & Processes \\
\hline & Session 1
\end{tabular}

- $\quad$ To increase media literacy

- To reduce internalization of the cultura appearance ideal

- To educate on appearance-related conversations

- To highlight the impact of 'fat-talk' and teasing

- To develop skills for situations involving 'fat-talk' or teasing

- $\quad$ To reduce 'fat-talk' and appearance-related teasing

- To educate on body comparison

- To increase skills in identifying body comparison

- To develop skills for avoiding body comparison

- To revisit and recap the issues covered within the program
- What is the media?

- The different techniques that can be used by the media to manipulate media images (media images are not real)

- Appearance does not equal how valuable you are

- The ideal body' differs across time and between cultures

$$
\text { Session } 2
$$

- What are appearance-related conversations' and 'fat-talk'?

- Exploration of the strategies that could be used during situations that involve fattalk or appearance-related teasing
- Facilitator presentation followed by class discussion

- $\quad$ Film clip, group worksheets and class discussion

- Individual brainstorming and class discussion

- Handout - homework

- Facilitator presentation, class discussion

- Individual brainstorming, group role play, class discussion

\section{Session 3}

- Introduction to body comparison and its negative consequences

- Exploration of strategies that could be used instead of body comparisons (do not fall into the comparison trap)

- $\quad$ Emphasis on positive qualities not related to appearance

- Review sessio
- Facilitator presentation, class discussion

- Individual worksheets, class discussion

- Individual brainstorming and class activity

- Film clip, facilitator recap and question 
Running head: SCHOOL BODY IMAGE INTERVENTION

Table 3

Mean gender differences on study variables at baseline

\begin{tabular}{lcccccccc}
\hline & \multicolumn{2}{c}{ Girls } & & \multicolumn{2}{c}{ Boys } & & & \\
\cline { 2 - 3 } & Mean (SD) & & Mean $(S D)$ & $d f$ & $p$ & $\eta_{p}{ }^{2}$ \\
\cline { 2 - 3 } Body satisfaction (a) & $12.21(3.50)$ & & $13.50(4.01)$ & 86 & .14 & .03 \\
Cultural appearance internalization (b) & $17.50(5.38)$ & & $14.91(6.21)$ & 86 & $.04 *$ & .04 \\
Appearance-related conversation (b) & $12.93(5.62)$ & & $11.35(6.36)$ & 85 & .23 & .02 \\
Appearance-related comparison (b) & $13.87(3.88)$ & & $11.84(4.20)$ & 82 & $.02 *$ & .07 \\
Appearance-related teasing (b) & $43.80(9.95)$ & & $35.17(11.27)$ & 86 & $.01 *$ & .01 \\
Restrained eating (b) & $9.13(4.27)$ & & $7.89(3.98)$ & 83 & .17 & .02 \\
Emotional eating (b) & $5.79(2.88)$ & & $5.83(3.25)$ & 86 & .95 & .04 \\
Self-esteem (a) & $2.79(1.09)$ & & $3.50(1.26)$ & 86 & $.01 *$ & .08 \\
Intervention topic knowledge (a) & $140.5(3.53)$ & & $13.50(4.00)$ & 86 & .50 & .05 \\
\hline
\end{tabular}

Note. (a) Higher scores are more desirable; (b) Lower scores are more desirable; ${ }^{*}=p<.05 ; \eta_{p}{ }^{2}=$ partial eta squared. 
Running head: SCHOOL BODY IMAGE INTERVENTION

Table 4

Univariate time $x$ condition interactions (significance levels and effect sizes)

\begin{tabular}{lccc}
\hline & Pre-test to post-test Time X Condition interactions \\
\cline { 2 - 4 } Girls & $F(d f=1,30)$ & $p$ & $\eta_{p}{ }^{2}$ \\
\hline Body satisfaction & 12.90 & $.001^{*}$ & .30 \\
Cultural appearance internalization & 1.77 & .19 & .06 \\
Appearance-related conversation & 5.12 & $.031^{*}$ & .15 \\
Appearance-related comparison & 5.43 & $.027^{*}$ & .15 \\
Appearance-related teasing & 2.26 & .14 & .07 \\
Restrained eating & 4.83 & $.036^{*}$ & .14 \\
Emotional eating & 15.79 & $.001^{*}$ & .35 \\
Self-esteem & 0.27 & .61 & .01 \\
Intervention topic knowledge & 6.94 & $.013^{*}$ & .19 \\
\hline & Pre-test to post-test Time X Condition interactions \\
\cline { 2 - 4 } Boys & $F(d f=1,30)$ & $p$ & $\eta_{p}{ }^{2}$ \\
\hline Body satisfaction & 0.22 & .64 & .01 \\
Cultural appearance internalization & 8.95 & $.005^{*}$ & .18 \\
Appearance-related conversation & 4.01 & $.05^{*}$ & .09 \\
Appearance-related comparison & 1.64 & .21 & .04 \\
Appearance-related teasing & 0.30 & .59 & .01 \\
Restrained eating & 0.54 & .47 & .01 \\
Emotional eating & 0.04 & .08 & .00 \\
Self-esteem & 0.02 & .89 & .00 \\
Intervention topic knowledge & 2.27 & .14 & .05 \\
\hline Note* & & &
\end{tabular}

Note. ${ }^{*}=p<.05 ; \eta_{p}{ }^{2}=$ partial eta squared. 